\title{
The Roles of Mass Media and Community Media in Multiple Great Disaster: From the Experience of Great East Japan Disaster in 2011
}

\author{
Kuniko Sakata Watanabe ${ }^{1}$, Akiko Ogawa ${ }^{2}$, Ayano Kubota ${ }^{3}$ \\ Graduate School of Information Sciences, Tohoku University, Japan ${ }^{1}$, \\ Graduate School of Informatics, Nagoya University, Japan²,3 \\ \{k-sakata@media.is.tohoku.ac.jp¹, a-ogawa@i.nagoya-u.ac.jp², ayano.kubota.t7@dc.tohoku.ac.jp $\left.{ }^{3}\right\}$
}

\begin{abstract}
This paper aims to clarify the roles of mass media as well as community media including temporary disaster radio stations in case of a serious and multiple disaster, based upon researches in relation to Great East Japan Disaster in 2011. For this purpose, firstly, the data of questionnaire to TV stations in Tokyo and those in three main disaster areas are analysed. Secondly, interview data of community FM broadcasters, including temporary disaster FM broadcasters of the disaster areas are examined. Thirdly, as a concrete example, "Voice of Fukushima," that is a remarkable activity for sending individuals" voices in Fukushima to the world by means of media, is discussed as a case study. After these discussions, it is clarified what was severe and what was lacked for each of media. In conclusion, important and alternative roles of media are reconsidered.
\end{abstract}

Keywords: Community Media; Disaster information; Fukushima; Great East Japan Disaster; Mass Media; Tohoku

\section{Introduction}

On March 11 in 2011, Japan unexpectedly experienced such a terrible multiple and widearea disaster. It is now called Great East Japan Disaster (hereinafter referred to as " 3.11 ") . It revealed large amount of problems in Japanese society in every field including rescue, evacuation, every kind of support, political judgment, economic disparity, community ties, and much more fields. However, one of the most important problems was that of information and media. The victims could not sufficiently get necessary information that mattered of life or death for a while after the earthquake and tsunami had attacked Tohoku area in Japan. In addition, they could have no way to tell and share their real nervous conditions and feelings to the world. This has not been so much changed even now. This paper attempts to discuss roles of mass media and community media when catastrophe unanticipatedly happens, based on the studies that have been conducted since 3.11. What is more important point is that this paper is written from the 'victim's' point of view. Two of the authors were in Tohoku when the disaster occurred and have continuously worked in the disaster area. They have made research as very few researchers in Tohoku disaster area.

First, the paper will clarify challenges and struggles of mass media, according to the results 
of questionnaires and interviews to some local and central TV stations including public broadcasting NHK as well as audiences in the disaster area. It could illustrate what mass media faced in the multiple great disaster and how they could or could not have overcome a large number of obstacles. In addition, what people actually expected and needed to them during and after 3.11. Based on these findings, the limitation of disaster information through mass media as well as its prospect will be argued. Second, efforts and problems of community and temporary disaster FM broadcasters in Fukushima will be discussed. Those broadcasters, particularly in Fukushima, played an essential role in helping relocated residents who become settled in temporary shelters, where it was still unknown whether they could return home due to the Fukushima No.1 Nuclear plant accident.

Third, the case of "Voice of Fukushima" will be introduced and analysed as a case study. There have been many activities in Tohoku area that have challenged such extremely difficult situation of disaster areas. "Voice of Fukushima" is one of highly evaluated activities in Fukushima. They have been trying not only to disseminate information to the world, but also to archive even trivial information and individual memories to succeed to the following generation. Finally, this paper will illustrate how it is possible/impossible to mediate and share personal. voices and thoughts of victims through various media in the world as well as in the future. Then, it is concluded what not only media but also "we" can do for ourselves as well as for others including (possible) victims, before, during and after an unexpected disaster.

\section{Method}

For the purpose of the above discussion, we applied the following methods. Firstly, in order to investigate the then situation of mass media, we made questionnaires and interviews to TV stations in the three main disaster areas, that are, Iwate, Miyagi and Fukushima prefectures as well as those in Tokyo as the centre of Japan. In contrast, we also made audience research in two small disaster areas. Namely, we made questionnaires and interviews to victims in Yamamoto Town in Miyagi and Namie Town in Fukushima. In addition to these 'sender' as well as 'audience' researches, we also attempt to make content analysis with the use of TV progmme archives in National Institute of Informatics (NII). Although we actually made this comprehensive research as a project, in this paper, we focus on the result of the questionnaire to TV stations with slight reference to another researches. We posted the questionnaire to 18 TV stations, including 4 in Iwate, 5 in Miyagi, 4 in Fukushima and 5 in Tokyo. Then we collected answers from 13 stations, including 2 in Iwate, 4 in Miyagi, 4 in Fukushima and 3 in Tokyo. The response rate is $72 \%$. The results of the above questionnaire are analysed below.

Secondly, in order to understand possibilities and problems of community FM broadcasters during and after the disaster, in-depth interviews with broadcasters conducted in 2017-18 were examined. In order to shed light on the struggles and possibilities of broadcasters, this paper particularly focuses on the interviews of three directors of community and temporary disaster FM broadcasters in Fukushima. Furthermore, in order to examine activities to overcome these problems concerning media environment, "Voice of Fukushima", which collects and broadcasts first-hand voices in Fukushima and disseminate them to the world, is introduced as one of the most remarkable cases.

\section{Results and Discussion}




\subsection{Experiences of TV stations in disaster area}

As the first question came from our sense of discomfort with media as a whole within the disaster area. Particularly TV coverage of victims and disaster areas strongly irritated us. Therefore, we decided to make a research in order to investigate the above 7 items. We had three main sensitive questions; (1) Who made coverages from whose point of views, (2) How deviation of information and coverage were produced depending on the areas, and (3) What are main factors for those problems.

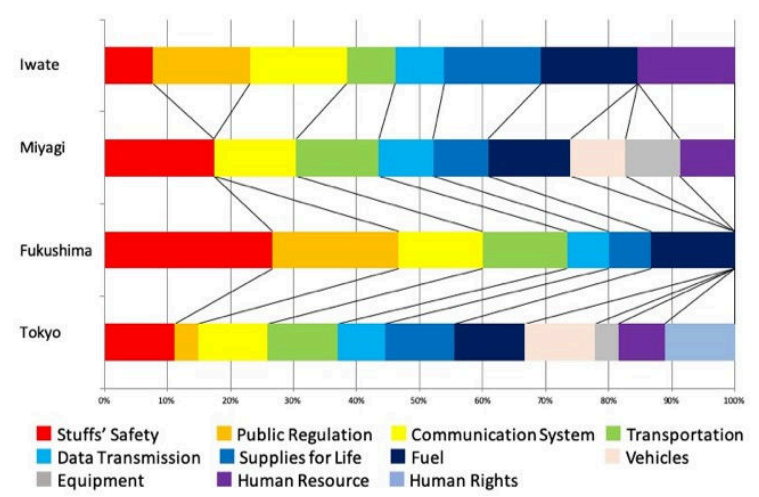

Fig 1. Obstacles for News Gathering and Reporting in Disaster Areas

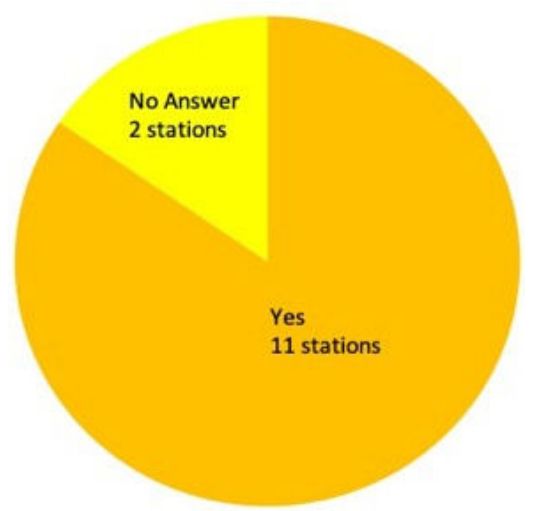

Fig 2. Existence of Areas Impossible to be Covered Despite its Importance

The followings are main results. Figure 1 demonstrates that TV stations had many kinds of obstacles for collecting information depending on the area. According to the previous question, it was proved that one hundred percent TV stations faced a lot of problems during news gathering in heavily damaged areas. Figure 1 also shows what are actually problems for them. These obstacles disabled them to enter dangerous areas, although there were yet a lot of people left behind.

According to Figure 2, it is evident that a lot of damaged areas could not be covered by them. This fact actually induced the second tragedy. Disaster areas that were not aired on TV could get much less relief goods and funds than those were aired. This was also proved by Miura (2012). 


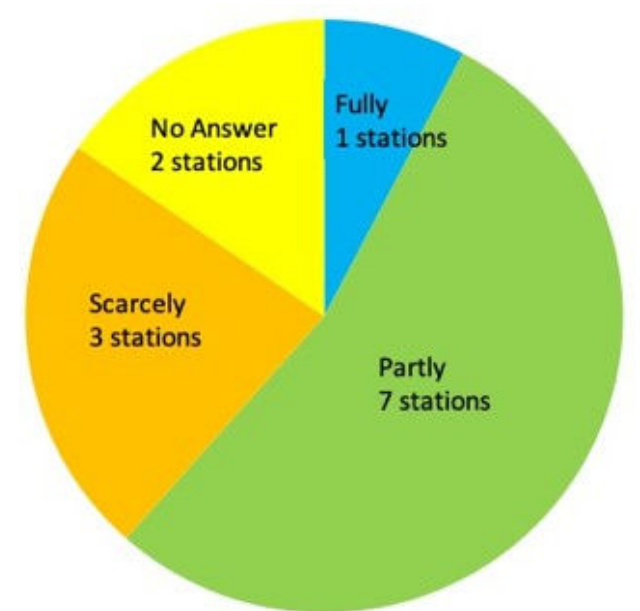

Fig 3. Usefulness of the Manuals for Data Collecting and Reporting Information in Case of Disaster

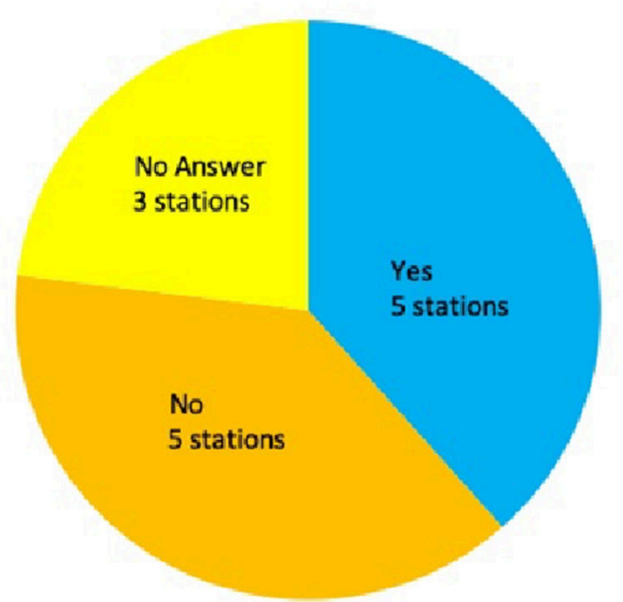

Fig 4. Existence of Reliable Information Source except Government and Administrative Division Reporting Information in Case of Disaster

Figure 3 shows if their existed manual for news gathering were useful. It can be hardly said that they were useful enough. Although it is not shown in the chart, what was lacked in case of multiple disaster, including earthquake, tsunami and diffusion of radioactivity, has been uncovered according to interviews to TV stations. In most of the cases, such a large-scaled disaster was never expected. Above all, the explosion of a nuclear powerplant was beyond our expectation. This is not only for us but also everybody in the world. In any case, we have never expected such a multiple disaster like 3.11 and, as a matter of course, have never been prepared to such the worst situation. Moreover, according to Figure 4, the half of them failed to find fully reliable information source except the government and administration in all such chaos. As a result, they could not report necessary news for us with confidence. 


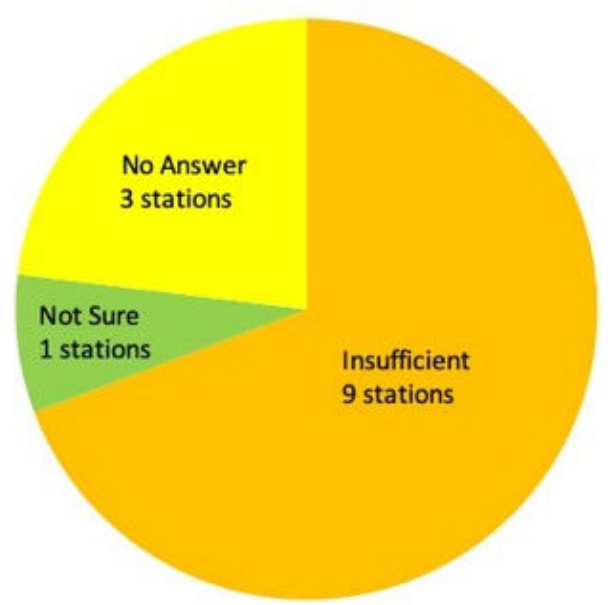

Fig 5. Sufficiency of Scientific Knowledge for Reporting of Nuclear Powerplant

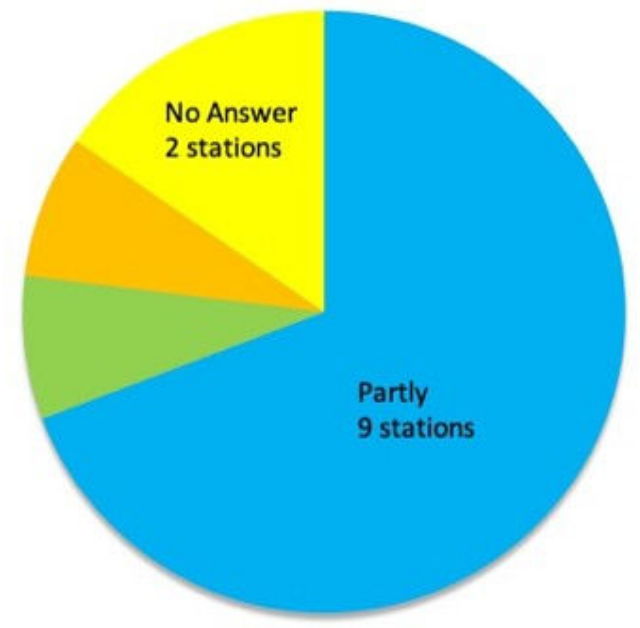

Fig 6. Capability of Reflecting Diverse Positions and Opinions

What is worse, they confessed their scientific knowledge was not far enough, according to Figure 5. In the interviews, some TV stations particularly in Fukushima, they were not sure if it was right that they had educated stuffs as a 'generalist.' They wondered if they should grow 'specialist' for future possible scientific incidents. Finally, at that time in Japan, people had no way to judge by themselves what was true or false through media including mass media, newspapers, the Internet. We could hardly have every kind of media literacy, as it was totally beyond a question of our 'literacy.' Figure 6 shows if TV programmes could reflect diverse opinions and give unbiased information to audiences. According to audience researches we conducted after that, people did less think that TV could success in this point than TV stuffs thought. There is undoubtedly a big gap between senders and audiences. We can find it is still open to question.

In this way, it cannot be said that TV could work for social requirements enough, as TV stations themselves admits that fact. If this is true, how could we have done for our lives. How could we get necessary information? How could we judge what we should do next? 


\subsection{The Difficulties of Community FM Broadcasters in Fukushima}

After 3.11, the Ministry of Internal affairs and Communication granted permission to 30 communities in the disaster area to operate emergency-disaster FM stations. In Fukushima, two existing community FM broadcasters applied for temporary disaster FM licenses. Four newly established stations in affected communities did the same. Particularly, FM Iwaki played an important role as the only local media outlet to provide information to citizens. Whilst mass media reporters were forced to evacuate by their organizations, FM Iwaki remained in the city. It also provided various information to people who were evacuated from the other towns within a $20-30 \mathrm{~km}$ radius from the power plant.

Odagaisama FM of Tomioka Town was launched in May 2011 in Koriyama, where most residents had been evacuated due to the incident and a temporary municipal office was located. This station was established not only to provide disaster information, but also to connect with townspeople evacuated nationwide through their municipal radio app. The station was managed by Tomioka Council Social Welfare staff, who had never previously participated in radio broadcasting. Yoshida, a member of staff, found that, what most helped the evacuees were moments of laughter, for example, when people heard radio announcers make mistakes or when sharing funny stories. The station also realized that the townspeople needed 'neighbour's chat' type of information in their programmes. The staff intentionally broadcasted in the Tomioka dialect to maintain a connection between relocated listeners and their hometown.

Hibari FM, a temporary disaster FM broadcaster, was established by Minamisoma City in April 2011. The city was divided into three types of evacuation zones: 1) evacuation zone within $20 \mathrm{~km}$ from the Fukushima No.1 nuclear power plant, 2) sheltering zone, and 3) zone with no instruction. As a result, residents in the evacuation zones were forced to relocate, while other residents were able to remain in their own houses. Under these circumstances, Hibari FM was established with the help of professional volunteers. One of the most important pieces of information was the monitoring data of the environmental radioactive level at 129 positions in the city. In their programme, a doctor of radiology broadcasted a programme explaining radiation effects to local people. It also broadcasted survivors' first-hand voices. They also broadcasted folk songs in their region for senior citizens who evacuated from their community. Hibari FM had been broadcasting for seven years. Its closure in March 2018 was lamented by community members.

In the first year, many programmes from these stations presented stories and voices of volunteers and residents who worked hard to overcome the disasters and their loss. These voices and stories struck a chord with the residents, with feeling a bond between people. They also broadcasted essential information such as radiation levels, and compensation procedures to their listeners. However, as the evacuation zones which determined varying levels of compensation were decided, divisions among residents arose. The compensation for evacuations caused by the nuclear incident was much higher than for someone whose house was destroyed by tsunami. Such differences could easily break community ties. Staff members of those stations mentioned that the issue of compensation was taboo for broadcasting in the community. For Example, the staff member of Hibari FM said, 'We would not like to encourage such a division and confrontation. If a damage compensation suit was won, other people might complain about it. Thus, another difference could make another division.' It means that community FM broadcasters knew that the residents had negative feelings and experienced a difficult reality as 'we victims.' A director of FM Iwaki emphasized, 'We know that there would be no answer. We know it would not get better if we broadcast matters that divided us.' 
Unlike Tokyo-centred broadcasters, the staff of community FM broadcasters are also victims and community residents. For those professionals, it seems difficult to cover news stories that may broaden divisions among listeners or hurt residents. Community FM broadcasters have an advantage of circulating detailed local information in addition to generating community bonds among residents. However, they have a difficulty when they need to criticize community members, including authorities, or cover stories that may divide residents. Thus, marginalized voices are easily neglected, not only by mass media, but also by community media.

\subsection{Attempt and Dilemma of Voice of Fukushima}

As a general incorporated association, Voice of Fukushima was organised by producers of radio programme and practitioners of social education. After 3.11, Fukushima was at the mercy of not only government's handling for the nuclear accident but also, even more, coverage of mass media. As there are a lot of problems in themselves as discussed above, people in Fukushima got strong distrust on mass media. Under these circumstances, members of Voice of Fukushima thought, "people's living environment and their thoughts and feelings should be told in their own words." That is how, a radio programme "Voice of Fukushima" started in 2012.

Activities of Voice of Fukushima somewhat differs from any other media as its members regard themselves as "problem combatants who have a sight of transmitter" and they are working on practices to solve problems in the disaster area. While producing and transmitting radio programmes, they have conducted activities within Fukushima Prefecture according to changing situation. They have also made use of 'their own' experience as staff members at Odagaisama FM as above. Their experience is a cycle including interview, activity, then interview again and activity again and again. Finally the members become to realise their media are totally different from mass media through their activities in their community media. They also come to note that "it is not enough if we do the same things as mass media" and try to balance just like practitioners of local activity. In this respect, Voice of Fukushima totally differs from mass media and existing alternative media in Japan. It should be considered as a new form of media that was created under the special situation in Fukushima after 3.11.

The radio programme, "Voice of Fukushima" consists of interviews of local people who live under various circumstances in Fukushima. It was delivered to nine community radio stations for broadcasting in Japan once a week. The members of Voice of Fukushima travel around Fukushima in order to collect and transmit voices of local people living there to people in other prefectures. It made them know what 3.11 actually had caused for people there. At the same time, it gave them various knowledge in relation to their cultures and habits in Fukushima, where people have different opinions each other.

After 3.11, they made two major achievements in eight years. One is that the voices of people in Fukushima have been broadcasted on community radio stations outside the prefecture. Fukushima's 'present condition' and 'people's real voice' have been conveyed out to Fukushima. The second is that they have successfully developed human resources who could handle media by themselves. By bringing up 'media literacy' of young generation with people in local community, local residents also have become willing to transmit local information by themselves. However, it should be note that diminution of peoples' concern in the Fukushima problem is remarkable. It is still difficult for them to find a way to keep people concerned. Furthermore, it is too strong for children to wipe away the widespread images of Fukushima constructed through media confusion and after 3.11. 


\section{Conclusion}

As discussed above, even though every kind of media is expected to give necessary information for victims for surviving as well as just for continuing their everyday lives, depending on their role or function in society, there is no question that it was extremely difficult to overcome unexpected troubles and obstacles particularly during and after a multiple disaster. Not only mass media but also community media had experienced difficulties and limitation in dealing with their tragedies and difficulties. Even the latter had hesitancy to broadcast their real voices, including compensation problems, in order to avoid division among community members. Thus, as a result, the broadcasters collected survivors' voices, but could not decide broadcasting some of them.

It is also true that disaster victims can hardly speak out for some reasons. It can also be found in similar cases, for example, that of Hiroshima and Auschwitz. Seven and half years have passed since 3.11. It may not be enough time for some victims to tell their sadness, their pains, their regrets and feeling of loss, etc. However, as in the case of incidents during WWII such as Hiroshima and Auschwitz as above, some of the victims have now started to open their mouths after such long time. It may need more time for them to speak out than expected. After long time, after the wound has been healed, and probably after he/she have recognised that his/her words should be left to the next generation as a piece of important history.

Therefore, it should be noted what is important for media's role is not only giving information to people in any aspects, but also recording and archiving what they have experienced, what they felt and thought, and what was actually needed by victims. Media can record, preserve and give necessary information as well as victims' important words beyond time and space. This is probably alternative roles of media for possible disaster in future.

Otherwise, human will repeat such unbearable tragedy that we have experienced in Tohoku.

\section{References}

[1] A. Kleinman, V. Das, M. Lock, M. (Eds). Social Suffering. Oakland, CA: University of California Press, 1997.

[2] K.W. Sakata, T. Mimura, T. Hisaichi kara Kanageru 3.11 to TV (3.11 and TV thinking from the Disaster Area). Tokyo: San Paulo, 2016.

[3] N. Ouchi. Rinji Saigai FM to iu Media (Temporary Disaster FM). Tokyo: Seikyu-sha, 2018.

[4] S. Kumagai (Ed.). Minna no Tojisha Kenyu (Research by Parties Concerned for all). Tokyo: Kongo-shuppan, 2017.

[5] R. Solnit. A Paradise Built in Hell: The Extraordinary Communities That Arise in Disaster. New York: Viking Adult, 2009.

[6] T. Kanayama. Soteigai no saigai de motomeraretamono-community radio no yakuwari. In Research Group of Disaster and Community Radio (ed.), Chiisana Radio kyoku to Community no Saisei, pp.9-19. Shiga: Osumi shoten, 2014.

[7] G. Spivak, G. (1987). Can the Subaltern Speak? In Lawrence, G. (ed) Marxism and the Interpretation of Culture. Champaign: University of Illinois Press, 1987.

[8] S. Miura. 311 Johogaku no Kokoromi: New Hodo no Data Bubunseki Kara (311 Informatics Trial: From Data Analysis of News Coverages). Tokyo: Iwanami-shoten, 2012.

[9] S. Matsuura. Column 1 Community hoso de Genpatsu ha ikani katarareteiruka. In 
Matsuura, S. (ed.), Nihon no community hoso: Riso to genjitu no hazama de, pp.45-51. Kyoto: Koyo shobo, 2017.

[10] T. Murakami. Consideration of peace education to pass down the fourth generation (the next generation). Hiroshima peace science, pp.33-50, 2008.

[11] NHK Broadcasting Culture Research Institute. "Higashi-Nihon Daishinsai Hasseikara 72 Jikan TV ga Tsutaeta Joho no Suii: Zaikyoku 3 kyoku no Hodo Naiyo Bunseki Kara (Transition of TV Information for 72 hours after Occurrence of Great East Japan Disaster: Content Analysis of 3 of TV stations in Tokyo)." Hoso Kenkyu to Chosa, March, 2-21, 2012.

[12] K. Sakata. "Higashi-Nihon Daishinsai kara Kangaeru Media to Subalternity (Media and Subalternity Thinking from Great East Japan Disaster)." Mass Communication Research, No. 82, pp. 67- 87, 2013.

[13] H. Ichimura. "Higashi nihon daishinsai go 27 kyoku tannjo shita rinji saigai housoukyoku no genjo to kadai (The Current State and Challenges: Temporary Radio Broadcasting Stations for the Great East Japan Earthquake Disaster)." Nihon no chiiki shakai to media, Kansai University Seiji- Keizai Kenkyujo, pp.115-146, 2012. 